\title{
Series-Based Solution for Analysis of Simply Supported Rectangular Thin Plate with Internal Rigid Supports
}

\begin{abstract}
Abubakr E. S. Musa and Husain J. Al-Gahtani
King Fahd University of Petroleum \& Minerals, Dhahran 31261, Saudi Arabia

Correspondence should be addressed to Abubakr E. S. Musa; bakri083@gmail.com

Received 6 October 2016; Revised 24 November 2016; Accepted 12 December 2016; Published 4 January 2017

Academic Editor: Luigi Di Sarno

Copyright ( 2017 A. E. S. Musa and H. J. Al-Gahtani. This is an open access article distributed under the Creative Commons Attribution License, which permits unrestricted use, distribution, and reproduction in any medium, provided the original work is properly cited.

In this study, Navier's solution for the analysis of simply supported rectangular plates is extended to consider rigid internal supports. The proposed method offers a more accurate solution for the bending moment at the critical section and therefore serves as a better analytical solution for design purposes. To model the plate-support interaction, the patched areas representing the contact between the plate and supports are divided into groups of cells. The unknown internal reactions at the centers of the divided cells are obtained by satisfying the compatibility conditions at the centers of the cells. Three numerical examples are presented to demonstrate the accuracy of the proposed analytical solution. The given examples reveal good agreements with those obtained by the finite element analysis. In addition, they show the advantage of the new solution as compared to the existing analytical solution which inaccurately estimates the location and magnitude of the maximum bending moment.
\end{abstract}

\section{Introduction}

Most of the international codes for the design of concrete structures give outlines for the design of slabs with regular layouts of internal supports. Availability of irregular layout and shapes of the internal supports is very common in engineering practice. Simply supported edges and internal supports can be found in common cases such as flat slabs with spandrel beams and load bearing structures with internal supports as shown in Figure 1.

The series-based solution is one of the most common early established methods for the analysis of plates, but less effort has been given to the analysis of internally supported plates. These series-based solutions have been well summarized in [1] based on Navier's and Levy's methods. Analysis of internally supported plates has also been covered in [1] for the assumptions that the layout is regular and the columns are to be treated as point reactions. This later assumption gives an infinite bending moment at the point of the support leading to an approximate procedure to treat the singularity of the bending moment at the center of the support as explained in [2]. This approximation, however, leads to a maximum bending moment at the center of the support instead of the face of the support as proven here by both proposed and finite element solutions.

Numerical methods, on the other hand, give more flexibility for the layout of the internal supports and the supporting conditions of the plate edge. The finite element method (FEM) is probably the most widely used method for solving a wide range of problems in structural engineering as demonstrated by the availability of numerous commercial software packages. One of the recent numerical methods attracting interests of the research community is the boundary element method (BEM), which has been used by many researchers to solve the present problem [3-11]. In these studies, different plate shapes, different shapes of the internal supports, and different boundary conditions have been considered. The direct boundary element method (DBEM) has been used in [12] for the analysis of complete frame consisting of a floor slab, walls, and columns with flexible and rigid conditions. A coupling of flexibility and boundary element methods has been employed in [13] to account for flexibility of the internal 


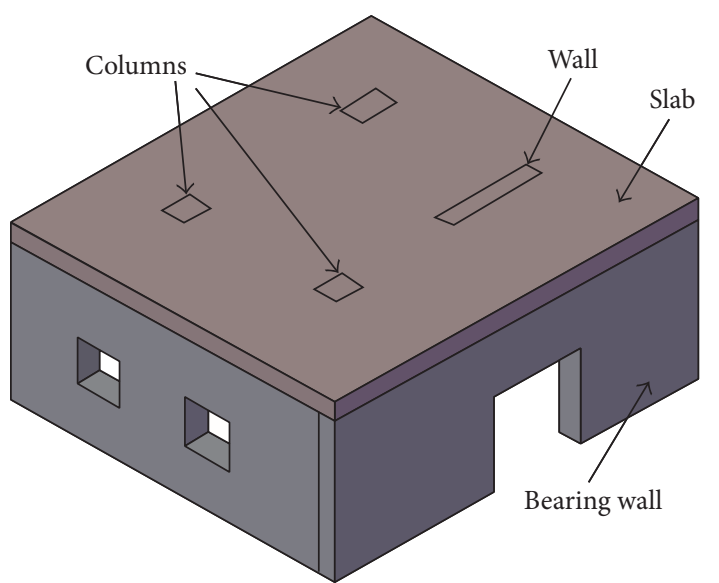

FIGURE 1: Load bearing structure containing floor slab with internal support.

supports. A special BEM method was used in [14] for the analysis of building floor slabs. In this method, the columns are assumed to remain flat under loading conditions.

The existing Navier solution is restricted to simply supported plates without internal supports. Introducing the internal supports makes the problem statically indeterminate to a degree equal to the number of internal supports and the analytical solution becomes too difficult to obtain. In order to accurately model the plate-internal support interaction, the plate-support contact areas are divided into groups of cells. The procedure leads to internal reactions equal in number to that of the divided cells. The reactions are then determined by satisfying the zero deflection at the centers of the cells. The results of the proposed method show a reasonable agreement with the FEM results.

\section{Formulation}

In the first part of this section, the formulation of Navier's solution is presented. The formulation leading to the solution of the reactions at the internal supports and hence the complete solution is discussed in the second part.

2.1. The Plate Problem. Consider a simply supported rectangular plate subjected to a distributed load $q(x, y)$ as shown in Figure 2.

The general differential equation is given by

$$
\nabla^{4} w=\frac{q(x, y)}{D}
$$

where $w$ is the deflection of the plate, $q$ is the applied load, and $D$ is the flexural rigidity of the plate. The solution of the above biharmonic differential equation requires two boundary conditions per each side of the plate. It is common in building frames to use flat plates with spandrel beams and internal supports or in load bearing structures to have a flat plate with internal columns or walls as shown in Figure 1. Therefore the deflection of the plate can be assumed to be zero at its boundary. Furthermore, the plate is allowed to rotate about an axis parallel to each side; that is, the plate has no resistance for

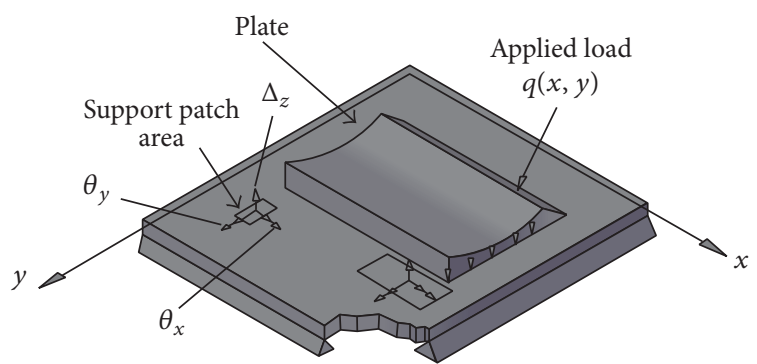

FIGURE 2: Simply supported rectangular plate with internal supports.

normal bending along each side. These boundary conditions can be expressed mathematically by the following equations:

$$
\begin{aligned}
& w=0, \\
& \frac{d^{2} w}{d x^{2}}=0 \\
& w=0, \quad(x=0, \quad x=a) \\
& \frac{d^{2} w}{d y^{2}}=0 \\
& \quad(y=0, \quad y=b),
\end{aligned}
$$

where $a$ and $b$ are the side length of the plate along $x$ and $y$, respectively, as shown in Figure 3. The load $q(x, y)$ can be expanded using Fourier's series as follows:

$$
q(x, y)=\sum_{m=1}^{\infty} \sum_{n=1}^{\infty} q_{m n} \sin \left(\frac{m \pi x}{a}\right) \sin \left(\frac{n \pi y}{b}\right),
$$

where the amplitude $q_{m n}$ is given by

$$
q_{m n}=\frac{4}{a b} \int_{0}^{b} \int_{0}^{a} q \sin \left(\frac{m \pi x}{a}\right) \sin \left(\frac{n \pi y}{b}\right) .
$$

Similarly, the deflection $w_{q}$ due to applied load $q(x, y)$ can be approximated by

$$
w_{q}(x, y)=\sum_{m=1}^{\infty} \sum_{n=1}^{\infty} w_{m n} \sin \left(\frac{m \pi x}{a}\right) \sin \left(\frac{n \pi y}{b}\right) .
$$

Substitution for the approximated $w_{q}$ given by (5) in the general differential equation given by (1) along with (3) and (4) yields an expression for the amplitude of the deflection:

$$
w_{m n}=\frac{q_{m n}}{\pi^{4} D\left[(m / a)^{2}+(n / b)^{2}\right]^{2}} .
$$

2.2. Plate-Support Interaction. Since the material is assumed to be linearly elastic, the superposition principle can be used to obtain the solution of the plate due to applied load $q(x, y)$ and the reactions from the internal supports. The internal 


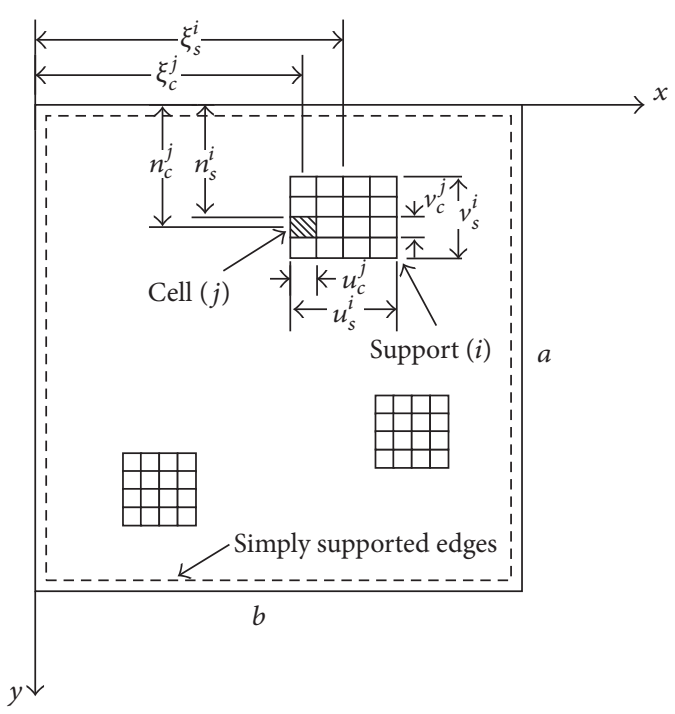

FIGURE 3: Dividing of the support to cells in a simply supported rectangular plate.

supports are modeled using the actual patched area of the support in order to accurately satisfy the compatibility of the deflection between the plate and the support. The patched area is divided to cells assuming that the reaction over each cell is distributed uniformly over the area of the cell and the deflection vanishes at the center of each cell.

Dividing the support $(i)$ into a group of cells $\left(n_{c}^{i}\right)$ produces a group of reaction forces $R_{c}^{j}\left(j, 1, n_{c}^{i}\right)$ over the cells of the support $(i)$ as shown in Figure 3 . These reaction forces produce a uniform stress $q_{r}^{j}$ over the area of each cell as represented by

$$
q_{r}^{j}=\frac{R_{c}^{j}}{u_{c}^{j} v_{c}^{j}}, \quad\left(j, 1, n_{c}\right)
$$

where $u_{c}^{j}$ and $v_{c}^{j}$ are the sizes of the cell area; $n_{c}$ is the total number of the cells of the internal supports. Expanding $q_{r}^{j}$ using Fourier's series

$$
q_{r}^{j}(x, y)=\sum_{m=1}^{\infty} \sum_{n=1}^{\infty} q_{m n r}^{j} \sin \left(\frac{m \pi x}{a}\right) \sin \left(\frac{n \pi y}{b}\right) .
$$

Following the same standard procedure, the amplitude of the cell load can be obtained from

$$
q_{m n r}^{j}=\frac{4}{a b} \int_{\eta_{c}^{j}-v_{c}^{j} / 2}^{\eta_{c}^{j}+v_{c}^{j} / 2} \int_{\xi_{c}^{j}-u_{c}^{j} / 2}^{\xi_{c}^{j}+u_{c}^{j} / 2} q_{r}^{j} \sin \left(\frac{m \pi x}{a}\right) \sin \left(\frac{n \pi y}{b}\right) .
$$

Using (7) in (9) and carrying out the integration, we get

$$
\begin{aligned}
q_{m n r}^{j}= & \frac{16 R_{c}^{j}}{\pi^{2} m n u_{c}^{j} v_{c}^{j}} \sin \left(\frac{m \pi \xi_{c}^{j}}{a}\right) \sin \left(\frac{n \pi \eta_{c}^{j}}{b}\right) \\
& \cdot \sin \left(\frac{m \pi u_{c}^{j}}{2 a}\right) \sin \left(\frac{n \pi v_{c}^{j}}{2 b}\right) .
\end{aligned}
$$

The amplitude of the defection due to the pressure $q_{r}^{j}$ can be obtained by the replacement of $q_{m n}$ in (6) by $q_{m n r}^{j}$; that is,

$$
w_{m n r}^{j}=\frac{q_{m n r}^{j}}{\pi^{4} D\left[(m / a)^{2}+(n / b)^{2}\right]^{2}} .
$$

So the deflection due to the pressure $q_{r}^{j}$ is given by

$$
w_{r}^{j}(x, y)=\sum_{m=1}^{\infty} \sum_{n=1}^{\infty} w_{m n r}^{j} \sin \left(\frac{m \pi x}{a}\right) \sin \left(\frac{n \pi y}{b}\right) .
$$

The total deflection can be obtained by superimposing the deflections due to the distributed load $q(x, y)$ and the cell reaction forces $q_{m n r}^{j}$; that is,

$$
w(x, y)=w_{q}+\sum_{j=1}^{n_{c}} w_{r}^{j} .
$$

Equation (13) involves the unknowns $R_{c}^{j}, j=1, n_{c}$, which can be determined by satisfying the zero-deflection condition at the centers of the cells; that is,

$$
w\left(\xi_{c}^{j}, \eta_{c}^{j}\right)=0, \quad\left(j, 1, \ldots, n_{c}\right) .
$$

Once (14) is solved for the support reactions $R_{c}^{j}$, the complete solution can be obtained from (13). A convergence analysis is needed to decide on the number of the terms after which the series solution given by (13) is truncated. The convergence analysis has been carried out in this study for all examples. However, the convergence results have been presented for Example 1 only.

\section{Numerical Examples}

Application of the proposed method is illustrated through the following three examples. The results are compared with those obtained by the finite element method (FEM) and the approximate solution available in [1].

Example 1 (simply supported square plate with one rigid column). A square plate with one square column as shown in Figure 4 is selected to study the convergence of the solution for both the number of series terms and the number of cells employed. The column is considered to be infinitely rigid at the zone of plate-column contact. The Poison ratio is $v=0.3$ and the size of the column is $u_{s}=v_{s}=a / 10$.

To study the series convergence, different number of terms in the series are selected as shown in Tables 1 and 2. As represented by bolded results in Table 1, the deflection $w$ converges very quickly with lower number of cells per the support but converges with a slower rate when more cells are used to model the column. This is mainly due to the fact that approximation of a patched load over a very small area using Fourier's series requires a large number of terms to get closer to the exact load function.

A similar trend but with a slower convergence rate is observed for the bending moment solution as shown in Table 2 . 
TABLE 1: Values of $w /\left(q a^{4} / D\right)$, at $x=a / 4, y=b / 4$.

\begin{tabular}{|c|c|c|c|c|c|}
\hline Number of terms & One-cell model & 4-cell model & 9-cell model & 16-cell model & 25-cell model \\
\hline 5 & 0.000424 & 0.000417 & 0.000142 & 0.000141 & 0.000061 \\
\hline 15 & 0.000439 & 0.000429 & 0.000343 & 0.000340 & 0.000284 \\
\hline 25 & 0.000439 & 0.000429 & 0.000376 & 0.000369 & 0.000337 \\
\hline 35 & 0.000439 & 0.000429 & 0.000383 & 0.000373 & 0.000355 \\
\hline 45 & 0.000439 & 0.000429 & 0.000383 & 0.000374 & 0.000362 \\
\hline 55 & 0.000439 & 0.000429 & 0.000383 & 0.000374 & 0.000365 \\
\hline 65 & 0.000439 & 0.000429 & 0.000383 & 0.000374 & 0.000365 \\
\hline
\end{tabular}

TABLE 2: Values of $M_{x} /\left(q a^{2}\right)$, at $x=a / 4, y=b / 4$.

\begin{tabular}{|c|c|c|c|c|c|}
\hline Number of terms & One-cell model & 4-cell model & 9-cell model & 16-cell model & 25-cell model \\
\hline 5 & 0.012638 & 0.012568 & 0.002850 & 0.002813 & 0.000669 \\
\hline 15 & 0.013146 & 0.013044 & 0.011620 & 0.011549 & 0.009772 \\
\hline 25 & 0.013146 & 0.013044 & 0.012041 & 0.011930 & 0.011415 \\
\hline 35 & 0.013146 & 0.013046 & 0.012311 & 0.012154 & 0.011879 \\
\hline 45 & 0.013146 & 0.013046 & 0.012323 & 0.012151 & 0.012002 \\
\hline 55 & 0.013146 & 0.013046 & 0.012323 & 0.012165 & 0.012009 \\
\hline 65 & 0.013146 & 0.013046 & 0.012323 & 0.012168 & 0.012011 \\
\hline 75 & 0.013146 & 0.013046 & 0.012323 & 0.012168 & 0.012012 \\
\hline 85 & 0.013146 & 0.013046 & 0.012322 & 0.012168 & 0.012012 \\
\hline
\end{tabular}

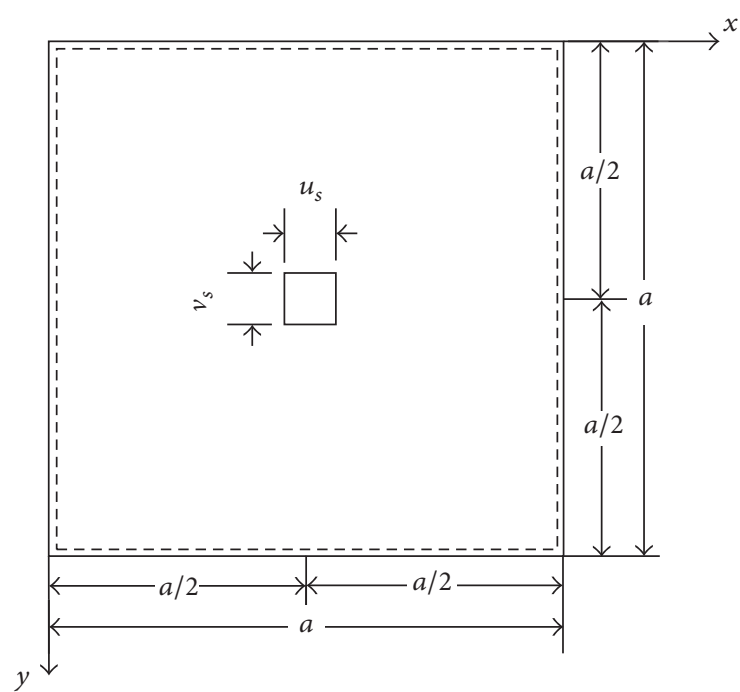

FIGURE 4: Simply supported rectangular plate with one central support.

In order to assess the results, a FEM model with 163220 domain elements and 1605 boundary elements has been used to solve the problem. Figures 5 and 6 compare the two solutions for the deflection $w$ and bending moment $M_{x}$, respectively. Figure 6 shows that at least 16 cells per support area have to be employed in order to satisfy the zerodeflection condition over all the patched area of the support with a reasonable accuracy.

The comparison for the bending moment results is given in Figure 6, which shows that an accurate estimate of the

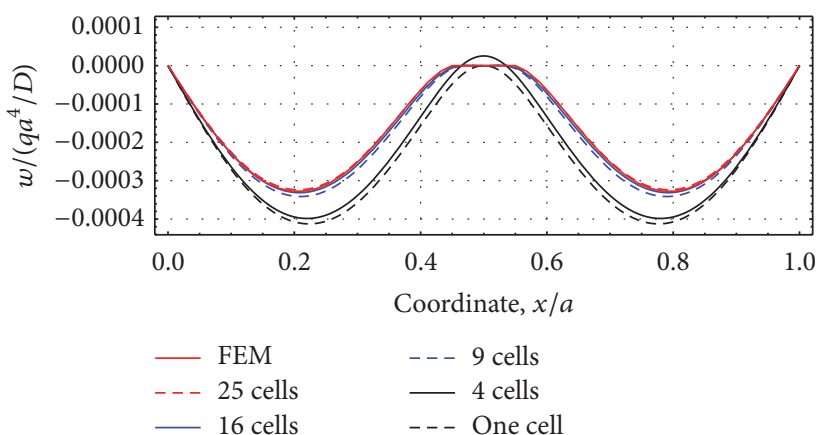

Figure 5: The deflection, $w$ at $y=a / 2$.

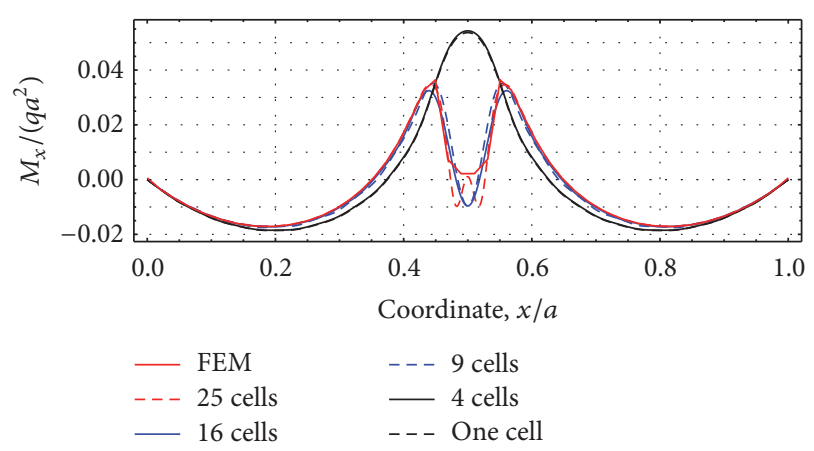

FIGURE 6: The bending moment $M_{x}$ at $y=a / 2$.

bending moment at the support face can be obtained even with a very small number of cells. The same cannot be said of the results at the center of the support. This is due to the 
TABLE 3: $\beta$ for the bending moment coefficient, $M=\beta q a^{2}$.

\begin{tabular}{|c|c|c|c|c|c|c|c|c|c|c|}
\hline \multirow[t]{2}{*}{ Point } & \multicolumn{2}{|c|}{ Proposed } & \multicolumn{2}{|c|}{ Available in [1] } & \multicolumn{2}{|c|}{ FEM } & \multicolumn{2}{|c|}{$\begin{array}{c}\text { \% difference between } \\
{[1] \text { and FEM }}\end{array}$} & \multicolumn{2}{|c|}{$\begin{array}{l}\% \text { difference between } \\
\text { proposed and FEM }\end{array}$} \\
\hline & $M_{x}$ & $M_{y}$ & $M_{x}$ & $M_{y}$ & $M_{x}$ & $M_{y}$ & $M_{x}$ & $M_{y}$ & $M_{x}$ & $M_{y}$ \\
\hline 1 & 0.017 & 0.017 & 0.021 & 0.021 & 0.017 & 0.017 & 23.5 & 23.5 & 0.0 & 0.0 \\
\hline 2 & -0.031 & 0.040 & -0.040 & 0.038 & -0.030 & 0.041 & 33.3 & -7.3 & 3.3 & -2.4 \\
\hline 3 & 0.068 & 0.023 & 0.069 & 0.025 & 0.068 & 0.023 & 1.5 & 8.7 & 0.0 & 0.0 \\
\hline 4 & 0.040 & -0.031 & 0.038 & -0.040 & 0.041 & -0.030 & -7.3 & 33.3 & -2.4 & 3.3 \\
\hline 5 & -0.138 & -0.138 & -0.140 & -0.140 & -0.138 & -0.138 & 1.4 & 1.4 & 0.0 & 0.0 \\
\hline 6 & 0.076 & 0.000 & 0.074 & -0.004 & 0.077 & 0.000 & -3.9 & - & -1.3 & 0.0 \\
\hline 7 & 0.023 & 0.068 & 0.025 & 0.069 & 0.023 & 0.068 & 8.7 & 1.5 & 0.0 & 0.0 \\
\hline 8 & 0.000 & 0.076 & -0.004 & 0.074 & 0.000 & 0.077 & - & -3.9 & 0.0 & -1.3 \\
\hline 9 & 0.052 & 0.052 & 0.053 & 0.053 & 0.053 & 0.053 & 0.0 & 0.0 & -1.9 & -1.9 \\
\hline
\end{tabular}

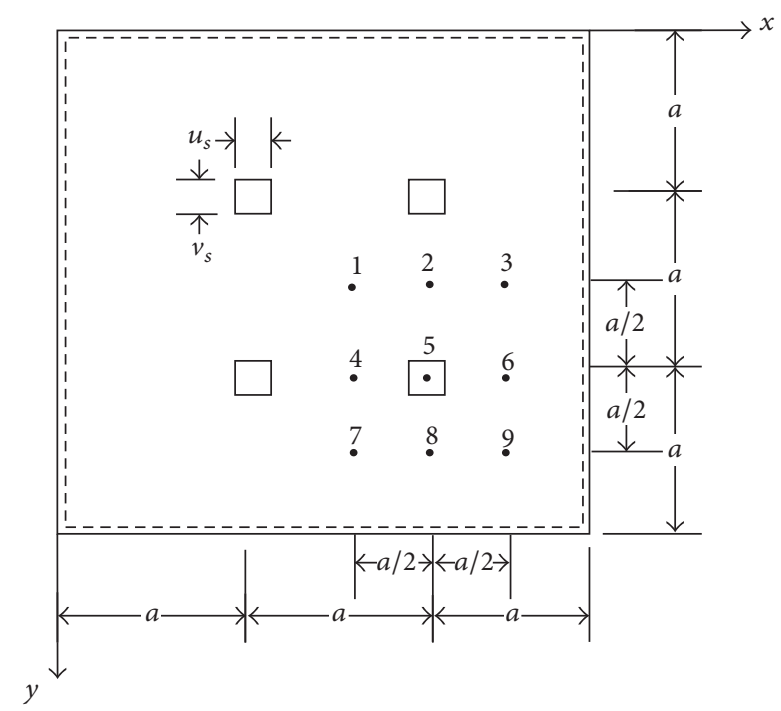

FIGURE 7: Simply supported rectangular plate with four internal supports.

fact that the assumption of the support being rigid requires too many cells in order to satisfy the zero deflection (and therefore, zero bending moment) at enough number of points within the support. This difficulty has been overcome in the FEM solution by using a very fine mesh at the support. It should be noted, however, that, in design practice, the critical bending moment, which is located at the face of the support, is more important and can be obtained very accurately by the proposed method with a 16 -cell discretization.

Example 2 (simply supported square plate with four point supported internal rigid columns). A simply supported square plate with four internal columns as shown in Figure 7 is subjected to a uniformly distributed load $q$. Poison's ratio is $v=0.2$ and the size of the column is $u_{s}=v_{s}=a / 4$.

In order to compare the results of this example with those given in Timoshenko's book [1], the plate is assumed to be restrained at a single point over each column and therefore,

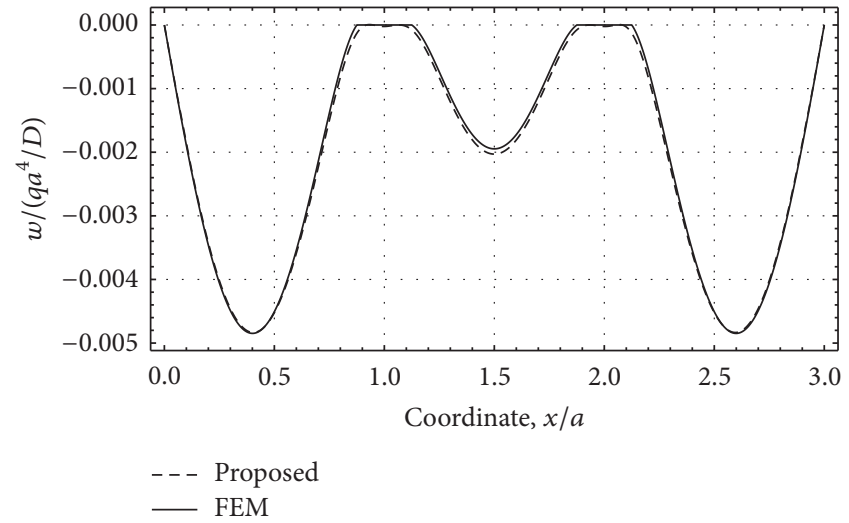

Figure 8: Deflection, $w$, along the line at $y=a$.

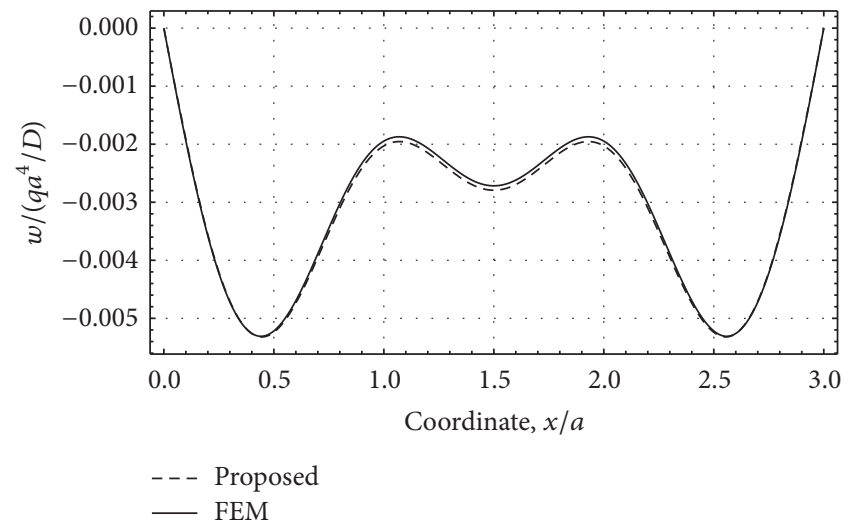

Figure 9: Deflection, $w$, along the line at $y=1.5 a$.

one cell is enough to apply the proposed method. Table 3 presents the results of the bending moment $M=\beta q a^{2}$ as obtained by the proposed method, the finite element method, and the approximate analytical solution [1].

The results clearly show that the results of the proposed analytical solution are much closer to those of the FEM solution with a maximum difference of $3.3 \%$. On the other hand, the results of the approximate analytical solution [1] 


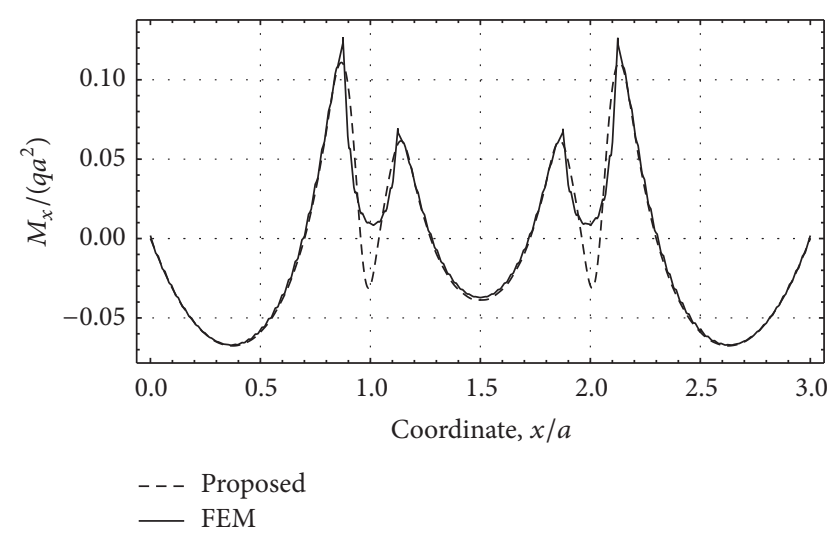

FIgURE 10: Bending moment $M_{x}$ at $y=a$.

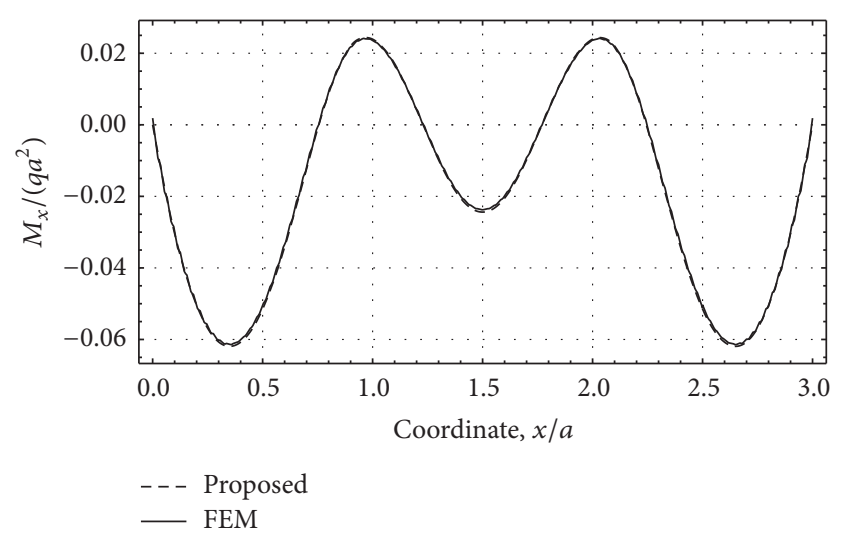

Figure 11: Bending moment $M_{x}$ at $y=1.5 a$.

deviate from the FEM solution for most of the points with a maximum difference of $33.3 \%$.

Example 3 (simply supported square plate with four internal rigid columns divided to cells). The same problem of Example 2 is resolved by forcing a uniform zero deflection over the whole patched area of each column. The results of the proposed method with 16 cells are compared with those of FEM in Figures 8-11.

Figures 8 and 9 show an excellent agreement between the present method and FEM for the deflection at the two sections of $y=a$ (through the columns centerlines) and $y=1.5 a$. For the bending moment $M_{x}$, however, Figure 10 shows that, at the section $y=a$, there is a good agreement between the two solutions at the face of the support and some variation at the middle of the support. This is attributed to the same reason explained for the results of Figure 6 in Example 1. As explained earlier, the agreement between the two solutions at the face of the support is more important since it represents the location of the maximum bending moment. The variation within the support can be improved by employing larger number of cells in the proposed method but from both practicality and economy viewpoints such effort is not justified. Figure 11 shows that there is an excellent agreement between the two solutions at a location away from the supports.

\section{Conclusions}

A simple analytical solution has been developed for the bending of simply supported rectangular plates with rigid internal supports. The solution is more accurate than the available analytical solution given in Timoshenko's book and can offer alternative method for the design of floor slabs. The results shown are in excellent agreement with the FEM at the critical locations, namely, the faces of supports. The deviation between the two solutions at the center of supports has no significance because the design codes are based on the maximum moment which is taken at the face of support/column. The proposed method can be easily extended to the solutions of plates with arbitrary layouts of the internal supports.

\section{Competing Interests}

The authors declare that they have no competing interests.

\section{Acknowledgments}

The authors gratefully acknowledge the support provided by King Fahd University of Petroleum \& Minerals and Saudi Aramco for this work.

\section{References}

[1] S. P. Timoshenko and S. Woinowsky-Krieger, Theory of Plates and Shells, McGraw-Hill, New York, NY, USA, 1959.

[2] R. Szilard, Theories and Applications of Plate Analysis, John Wiley \& Sons, Hoboken, NJ, USA, 2004.

[3] J. B. De Paiva, "Boundary element formulation of building slabs," Engineering Analysis with Boundary Elements, vol. 17, no. 2, pp. 105-110, 1996.

[4] C. J. de Oliveira, J. B. de Paiva, and Â. V. Mendonça, "Boundary element analysis of building slabs," in Proceedings of the World Congress on Engineering (WCE '15) 2015), vol. 2, pp. 1-6, London, UK, July 2015.

[5] G. R. Fernandes and W. S. Venturini, "Building floor analysis by the boundary element method," Computational Mechanics, vol. 35, no. 4, pp. 277-291, 2005.

[6] G. Bezine, "A boundary integral equation method for plate flexure with conditions inside the domain," International Journal for Numerical Methods in Engineering, vol. 17, no. 11, pp. 1647-1657, 1981.

[7] M. Guminiak and R. Sygulski, "The analysis of internally supported thin plates by the boundary element method. Part 1-static analysis," Foundations of Civil and Environmental Engineering, vol. 9, pp. 17-41, 2007.

[8] M. Guminiak and T. Jankowiak, "The analysis of internally supported thin plates by the boundary element method. Part 3initial stability analysis," Foundations of Civil and Environmental Engineering, no. 10, pp. 51-67, 2007.

[9] J. T. Katsikadelis, E. J. Sapountzakis, and E. G. Zorba, "A BEM approach to static and dynamic analysis of plates with internal supports," Computational Mechanics, vol. 7, no. 1, pp. 31-40, 1990. 
[10] M. Guminiak and K. Szajek, "Static analysis of circular and elliptic plates resting on internal flexible supports by the Boundary Element Method," Journal of Applied Mathematics and Computational Mechanics, vol. 13, no. 2, pp. 21-32, 2014.

[11] Y. F. Rashed, "Boundary element modelling of flat plate floors under vertical loading," International Journal for Numerical Methods in Engineering, vol. 62, no. 12, pp. 1606-1635, 2005.

[12] G. Hartley and A. Abdel-Akher, "Analysis of building frames," Journal of Structural Engineering, vol. 119, no. 2, pp. 468-483, 1993.

[13] Y. F. Rashed, "A coupled BEM-flexibility force method for bending analysis of internally supported plates," International Journal for Numerical Methods in Engineering, vol. 54, no. 10, pp. 14311457, 2002.

[14] L. De Oliveira Neto and J. B. De Paiva, "A special BEM for elastostatic analysis of building floor slabs on columns," Computers and Structures, vol. 81, no. 6, pp. 359-372, 2003. 


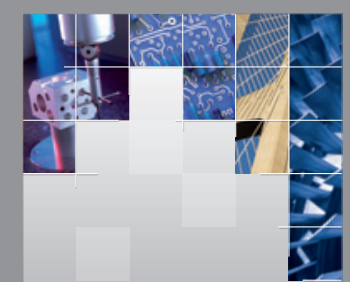

\section{Enfincering}
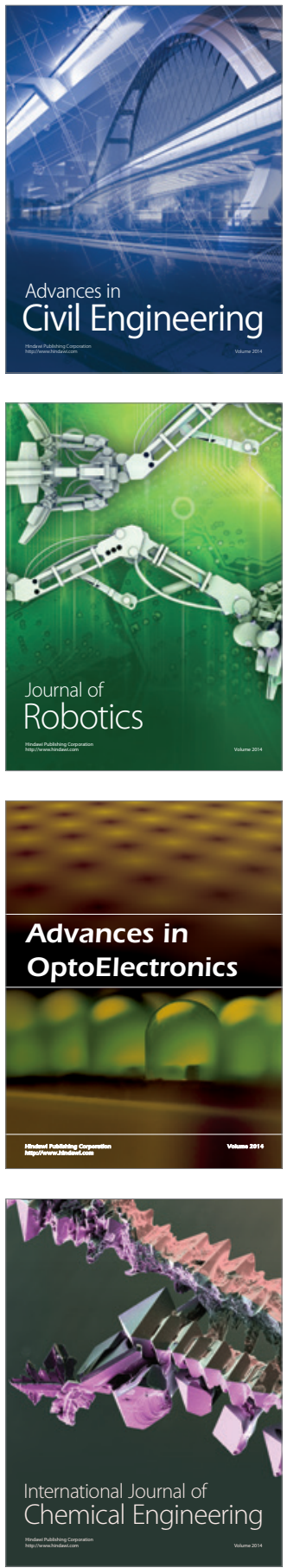

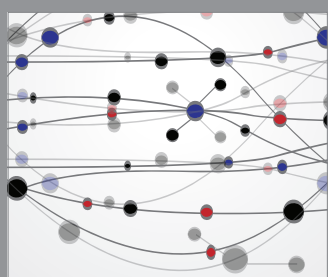

The Scientific World Journal

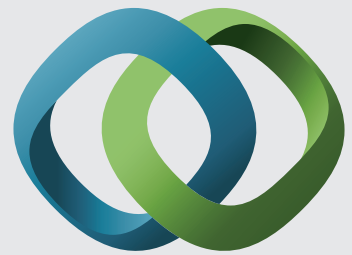

\section{Hindawi}

Submit your manuscripts at

https://www.hindawi.com
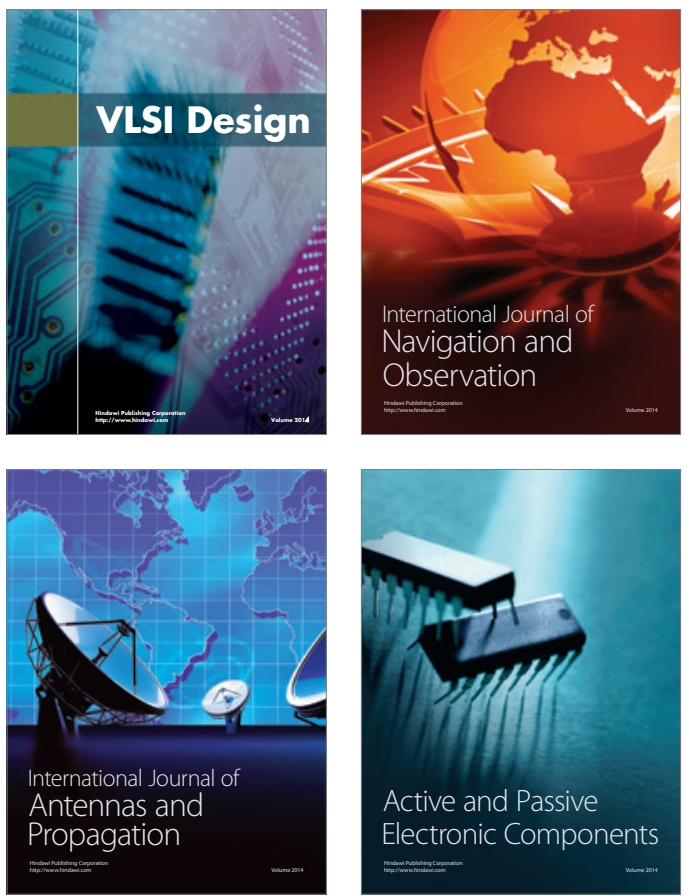
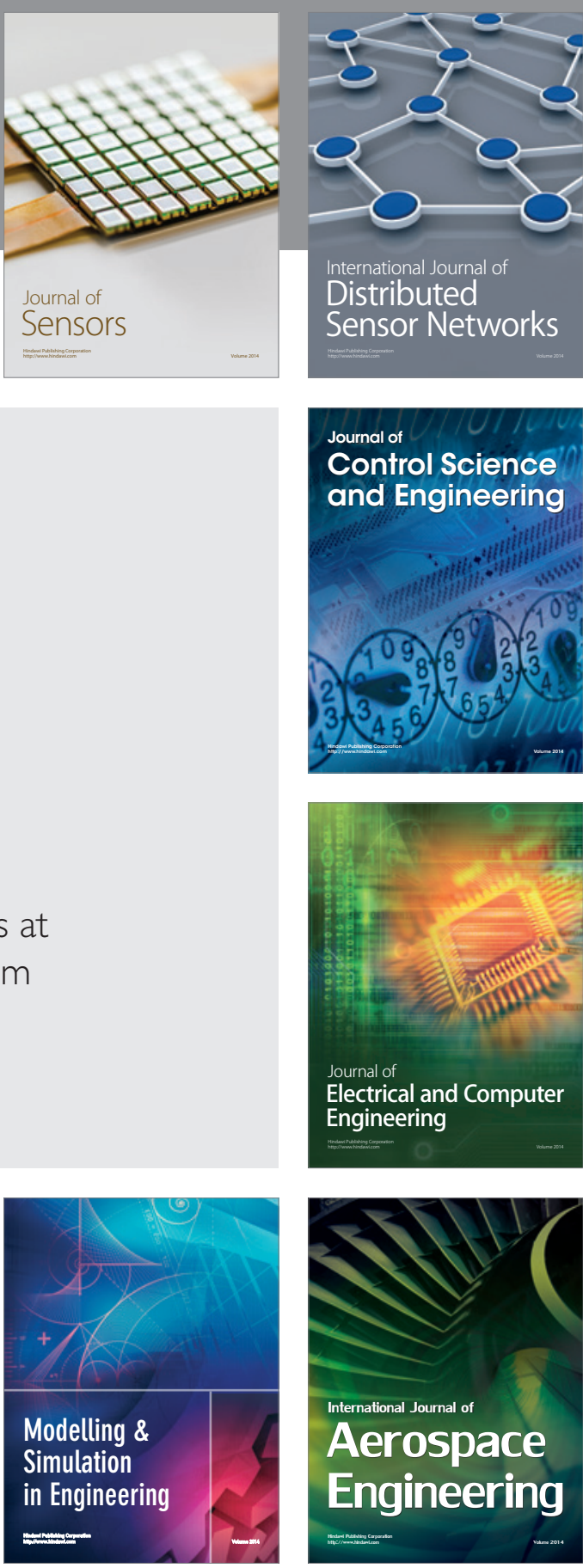

International Journal of

Distributed

Sensor Networks

$-$

Joumal of

Control Science

and Engineering
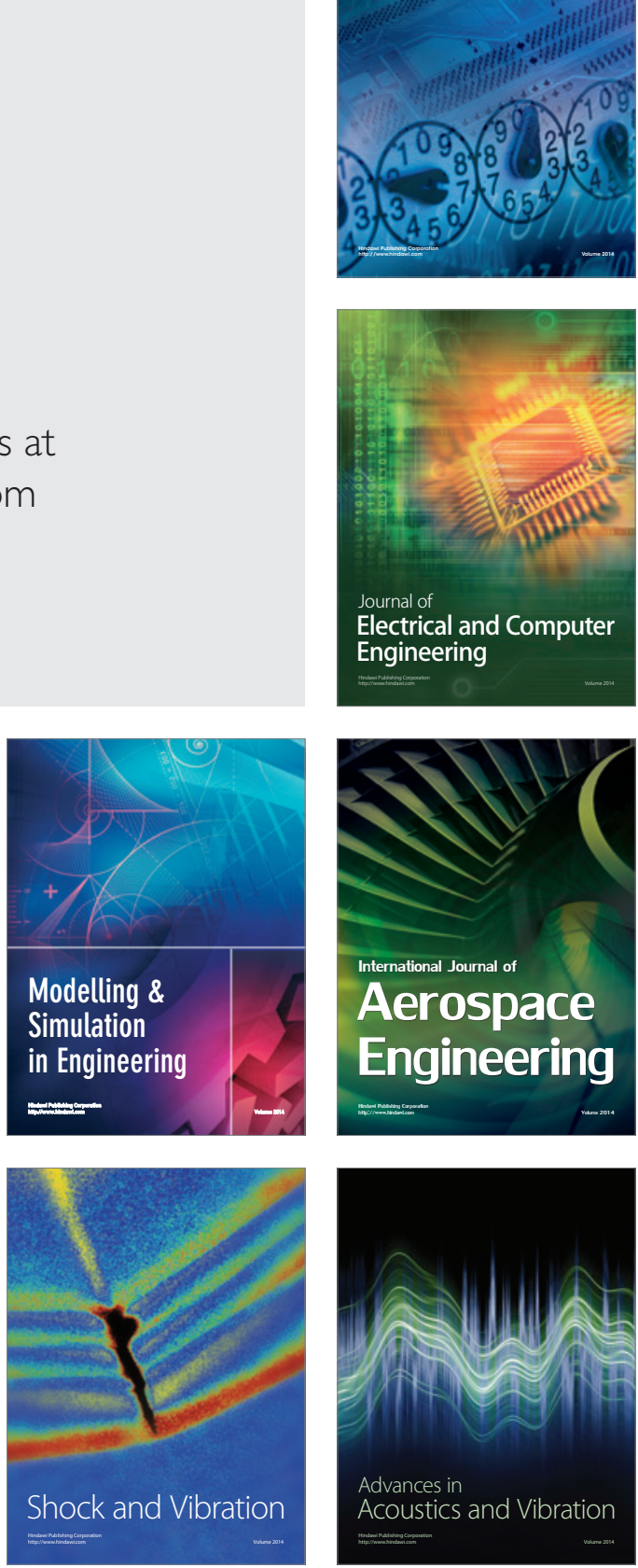